\title{
A new age and distance indicator of SNRs with nonthermal X-ray filaments
}

\author{
Aya Bamba ${ }^{1}$, Ryo Yamazaki $^{2}$ and Junko S. Hiraga ${ }^{3}$ \\ ${ }^{1}$ RIKEN (The Institute of Physical and Chemical Research) 2-1, Hirosawa, Wako, Saitama \\ 351-0198, Japan \\ email: bamba@crab.riken.jp \\ ${ }^{2}$ Department of Earth and Space Science, Graduate School of Science, Osaka University, \\ Toyonaka, Osaka 560-0043, Japan \\ email: ryo@vega.ess.sci.osaka-u.ac.jp \\ ${ }^{3}$ Department of High Energy Astrophysics Institute of Space and Astronautical Science (ISAS) \\ Japan Aerospace Exploration Agency (JAXA) 3-1-1 Yoshinodai, Sagamihara, Kanagawa \\ 229-8510, Japan \\ email: jhiraga@astro.isas.jaxa.jp
}

\begin{abstract}
It is discovered that young SNRs have very thin filaments emitting nonthermal Xrays from accelerated electrons. In this paper, a new age and distance indicator is proposed using the spectral and spatial features of nonthermal filaments. We applied this method to the Vela Jr. SNR, for which age and distance are still unknown, and estimated that this SNR is one of the nearest and youngest SNR in our Galaxy: the estimated distance and age are 0.33 $(0.26-0.50) \mathrm{kpc}$ and 660 (420-1400) years, respectively.
\end{abstract}

Keywords. methods: data analysis, supernova remnants, X-rays: individual (Vela Jr.).

\section{Introduction}

Recently, it was discovered that young SNRs have very thin filaments emitting nonthermal X-rays from accelerated electrons (Bamba et al. 2003), indicating that cosmic ray acceleration occurs efficiently in local regions, and as a result, the SNR age characterizes the spatial and spectral feature of filaments most effectively. Bamba et al. (2005) found the relation,

$$
\mathcal{B} \equiv \nu_{\text {roll }} / \theta_{d}^{2}=2.6_{-1.4}^{+1.2} \times 10^{27} t_{\text {age }}{ }^{-2.96_{-0.06}^{+0.11}} \mathrm{~Hz} \mathrm{pc}^{-2},
$$

where $\nu_{\text {roll }}, \theta_{d}, t_{\text {age }}$ are the roll-off frequency of synchrotron X-rays (Reynolds 1998), scale length in the downstream region of the shock, and the SNR age, respectively. In this paper, we make a method to estimate the distance and age of SNRs with $\mathcal{B}$, and apply it to a SNR, Vela Jr., with Chandra observations. This SNR is very curious because of a possible detection of $1.157 \mathrm{MeV} \gamma$-ray line emitted by ${ }^{44} \mathrm{Ti}$ (Iyudin et al. 1998). The other fact which makes this SNR famous is the existence of nonthermal X-rays from the rims (Slane et al. 2001), which implies that the rims of Vela Jr. are cosmic ray accelerators. However, nobody knows its distance and age. The details of the discussion are in Bamba et al. (2005b).

\section{Results}

Figure 1 are Chandra images of north-western (NW) shell of Vela Jr. We can see clear thin filaments in the hard band image, which is very similar to those in SN 1006. We chose three filaments, No. 1-3 in Figure 1(b) for the following analysis. The spatial and 


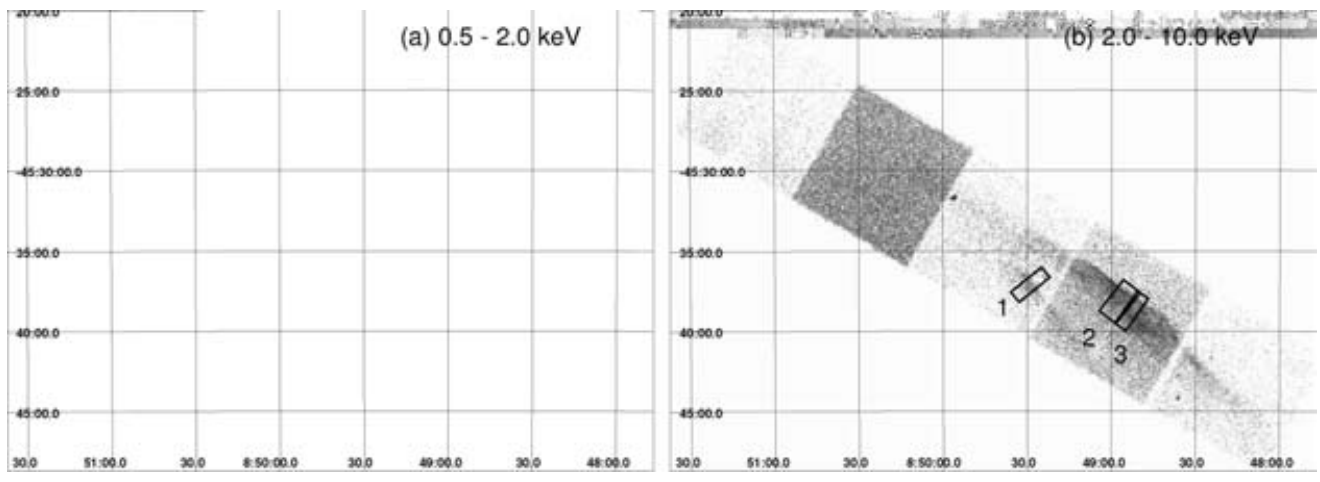

Figure 1. Chandra images of the NW rim of Vela Jr. in the $0.5-2.0 \mathrm{keV}$ band (a) and $2.0-10.0 \mathrm{keV}$ band (b).

Table 1. Spatial and spectral parameters of the filaments

\begin{tabular}{|c|c|c|c|}
\hline & 1 & 2 & 3 \\
\hline$\theta_{u}[\operatorname{arcsec}]$ & $19.0(12.1-31.4)$ & $3.68(2.69-5.75)$ & - \\
\hline$\theta_{d}[\operatorname{arcsec}]$. & $31.8(23.7-47.1)$ & $65.0(38.2-145)$ & $37.1(26.5-60.2)$ \\
\hline$\nu_{\text {roll }}\left[10^{16} \mathrm{~Hz}\right]$ & $3.7(23.7-47.1)$ & $5.3(3.6-7.3)$ & $4.3(3.4-5.3)$ \\
\hline Flux $_{2-10 \mathrm{keV}}\left[\mathrm{ergs}_{\mathrm{cm}}^{-2} \mathrm{~s}^{-1}\right] \ldots$ & $7.4 \times 10^{-13}$ & $1.8 \times 10^{-12}$ & $7.6 \times 10^{-13}$ \\
\hline
\end{tabular}

spectral analysis have been done in the same way to the SN 1006 case (Bamba et al. 2003), and we got the best-fit parameters as seen in Table 1. The spectral index at $1 \mathrm{GHz}$ is assumed to be 0.3 (Combi et al. 1999). The spectral feature indicates that the filaments emit synchrotron X-rays.

For the distance and age estimation, it needs another relation between the radius and age. We chose the SNR evolution model by Truelove \& McKee (1999) (see eq. (1) and (2), and Table 6 and 7 ), with the condition of constant interstellar medium of $0.1 \mathrm{~cm}^{-3}$, the ejecta mass of $1.4 M_{\odot}$, and the kinetic explosion energy of $10^{51}$ ergs. Together with the evolution model and the function $\mathcal{B}$, the distance and age are estimated to be 0.33 (0.26-0.50) kpc and 660 (420-1400) years, respectively. These results imply that Vela Jr. is one of the nearest and youngest SNRs in our Galaxy.

\section{References}

Bamba, A., Ueno, M., Koyama, K., \& Yamauchi, S. 2003, ApJ, 589, 253

Bamba, A., Yamazaki, R., Yoshida, T., Terasawa, T., \& Koyama, K. 2005, ApJ, 621, 793

Bamba, A., Yamazaki, R., \& Hiraga, J. S. 2005, ApJ, in press (astro-ph/0506331)

Combi, J. A., Romero, G. E., \& Benaglia, P. 1999, ApJL, 519, L177

Iyudin, A. F., et al. 1998, Nature, 396, 142

Reynolds, S. P. 1998, ApJ, 493, 375

Slane, P., Hughes, J. P., Edgar, R. J., Plucinsky, P. P., Miyata, E., Tsunemi, H., \& Aschenbach, B. $2001, A$ pJ, 548, 814

Truelove, J. K., \& McKee, C. F. 1999, ApJS, 120, 299 